\title{
Intravascular Ultrasound Correlates of Corrected TIMI Frame Count
}

\author{
Nihal ÖZDEMIR, ${ }^{1}$ MD, Cihangir KAYMAZ, ${ }^{1} \mathrm{MD}$, Cevat KIRMA, ${ }^{1} \mathrm{MD}$, \\ Murat AKÇAY, ${ }^{1} \mathrm{MD}$, Murat YÜCE, ${ }^{1} \mathrm{MD}$, Fikret TURAN, ${ }^{1} \mathrm{MD}$, \\ and Mehmet ÖZKAN, ${ }^{1} \mathrm{MD}$
}

\section{SUMMARY}

The TIMI frame count (TFC) is an index of coronary blood flow, and a correction in TFC (CTFC) for left anterior descending artery (LAD) has also been proposed. However, the relationship between TFC and intravascular ultrasound (IVUS) parameters of culprit coronary arteries has not been reported. The aim of this study was to investigate IVUSderived correlates of TFC before and after stenting, and to assess the validation of its correction for LAD. The study population was comprised of 38 patients with acute coronary syndrome or stable coronary artery disease studied by IVUS before and after stenting (LAD 21, circumflex 8, right coronary artery 9). For LAD, CTFC was calculated by dividing the TFC by 1.7. Preintervention luminal \% area stenosis was $82 \pm 12.3 \%$. Pre-and postintervention target lesion lumen areas were $1.8 \pm 0.5 \mathrm{~mm}^{2}$ and $8.5 \pm 0.5 \mathrm{~mm}^{2}$ $(P<0.0001)$, and CTFC were $35.3 \pm 16.8$ and $16.9 \pm 4.3(P<0.0001)$, respectively. In the 76 IVUS studies, CTFC showed a good correlation to luminal $\%$ area stenosis $(r=0.69$, $P<0.001)$, and a good and negative correlation to target lesion lumen area $(r=-0.70$, $P<0.001)$. Postprocedural improvement in CTFC showed a modest correlation to acute lumen gain $(r=0.5, P<0.05)$. With respect to culprit arteries, pre and postintervention IVUS parameters and CTFC, and net CTFC change after stenting were not different $(P>0.05)$. However, uncorrected TFC of LAD was significantly higher than both the CTFC of LAD and TFC of the other two coronary arteries $(P<0.05)$.

We conclude that CTFC is closely correlated to target lesion luminal area and luminal $\%$ area stenosis whereas a modest correlation is present between improvement in CTFC and acute luminal gain due to stenting. Results from different coronary arteries with comparable IVUS parameters seem to support the validity of a correction in TFC. (Jpn Heart J 2003; 44: 213-224)

Key words: TIMI frame count, Intravascular ultrasound, Stent

Over the last decade Thrombolysis in Myocardial Infarction (TIMI) flow grades have been widely used for the assessment of reperfusion in infarct-related arteries following thrombolytic or percutaneous intervention. ${ }^{1-8)}$ However,

From the ${ }^{1}$ Department of Cardiology, Kosuyolu Heart \& Research Hospital, Istanbul, Turkey.

Address for correspondence: Nihal Özdemir, MD, Department of Cardiology, Kosuyolu Heart and Research Hospital, Kadiköy, 81020, Istanbul, Turkey.

Received for publication February 21, 2002.

Revised and accepted July 25, 2002. 
because of several limitations associated with TIMI flow grade, new parameters that overcome the subjectivity and categorical nature of TIMI flow grade have been needed. The TIMI frame count (TFC) has been developed by Gibson, et al as a simple, reproducible, objective, and quantitative parameter of coronary flow assessed by angiography. ${ }^{2)}$ This method has been documented to be an accurate marker to assess angiographic success, and to predict long term patency, recovery of ventricular function, and clinical outcome. ${ }^{2-8)}$ A correction procedure for the left anterior descending artery by dividing the TFC by 1.7 has been proposed to provide an equivalency between different coronary artery territories with different anatomic length. ${ }^{2}$ Reported angiographic correlates of corrected TFC (CTFC) are based on two-dimensional luminographic measurements estimated from reference lumen and lesion diameters. However, the relationships between CTFC and cross-sectional luminal area and \% area stenosis measured by intravascular ultrasound (IVUS) have not been investigated.

The aim of the present study was to investigate IVUS-derived planimetric correlates of CTFC in patients in whom an intracoronary stent was implanted into the different culprit coronary arteries, and to assess the validation of the correction procedure for the left anterior descending artery with IVUS measures comparable to the left circumflex and right coronary arteries.

\section{METHODS}

The study population consisted of 38 patients ( 31 males, 7 females, mean age $56 \pm 5$ years) studied by IVUS before and after intracoronary stenting. The culprit coronary arteries stented were the left anterior descending artery in 21 patients, the circumflex in 8 , and the right coronary artery in 9 patients. Clinical presentation was acute coronary syndrome in 25 and stable coronary artery disease in 13 patients. The baseline characteristics, and angiographic and IVUSderived measurements are summarized in Table I. The protocol was approved by our hospital's institutional review board, and written informed consent was obtained from each patient before the procedures.

Angiographic study and corrected TIMI frame counting: Coronary arteriography was performed using an $8 \mathrm{~F}$ guiding catheter and non-ionic contrast medium (Hexabrix 320, Guerbet, France) by manual injection and was filmed at 12.5 frames/s (Siemens Coroscop T.D.P.). The angiographic diameters of the mean reference segments, minimal lesion diameters, and \% diameter stenosis between the different culprit arteries were comparable (Table I). Cineframes were counted by a frame counter (Tagarno A/S, Horsens, Denmark) according to the criteria for each territory defined by Gibson, et al. ${ }^{2)}$ An adjustment for all angiograms was performed by multiplying the TFC by a factor of 30 divided by the actual number 
(12.5 frames/s) of filming speed. For left anterior descending artery angiograms, a second correction (CTFC) was made by dividing the TFC by the 1.7 correction factor as proposed by Gibson, et al. ${ }^{2)}$

Definitions of the anatomic landmarks in TIMI frame counting: Frame counting was defined as the number of cineframes required for contrast to first reach standard distal anatomic landmarks. ${ }^{2)}$ The first frame used for TIMI frame counting was defined by a column of concentrated dye extending across $>70 \%$ of the origin of the arterial lumen with anterograde motion, and touching both borders of the origin of the artery, as reported previously. ${ }^{2}$ The last frame counted was defined as the frame in which dye first appears in the predefined distal landmark branches, but full opacification was not necessary. The following distal landmark branches were used for analysis: the distal bifurcation of the left anterior descending artery (ie, "mustache," "pitch-fork," or "whale's tail"); in the circumflex system, the distal bifurcation of the segment with the longest total distance that includes the culprit lesion; and in the right coronary artery, the first branch of the posterolateral artery. ${ }^{2)}$

Intravascular ultrasound imaging: Each patient was assessed by IVUS before and after coronary stent implantation. All patients were premedicated with aspirin and received heparin $(100 \mathrm{U} / \mathrm{kg})$ before the procedures. Intravascular ultrasound imaging was performed using a commercially available imaging system with a 30 $\mathrm{MHz}$ electronic transducer mounted on $3.2 \mathrm{~F}$ monorail ultrasound catheter (Endosonics Oracle in-vision imaging system 3.3). After the intracoronary administration of $200 \mu \mathrm{g}$ of nitroglycerin, the IVUS catheter was advanced over a 0.014 inch guidewire at least $1 \mathrm{~cm}$ distal to the target segment under fluoroscopic guidance. Using continuous pull-back, IVUS images were obtained and recorded on 0.5 inch s-VHS videotape for off-line quantitative analysis. Target lesion area was defined as the minimal lumen area measured during pull-back. Segments $\geq 1 \mathrm{~cm}$ proximal and distal to the target lesion, with cross-sectional area stenosis $<50 \%$ and no side branches, were defined as reference segments. ${ }^{9)}$ The vessel area inside the external elastic membrane and lumen area was manually traced. Planimetric measurements of proximal and distal reference segment area, and target lesion area were performed. ${ }^{10)}$ Mean reference vessel and lumen area, and luminal $\%$ area stenosis were calculated using a formula according to previously accepted definitions. ${ }^{10)}$

These formulas are given below: Mean reference lumen area $=[$ Proximal reference lumen area + Distal reference lumen area $/ 2$

Luminal $\%$ area stenosis $=[($ Mean reference lumen area - target lesion lumen area)/(Mean reference lumen area) $] \times 100$.

The intravascular ultrasound parameters are shown in Table I. All measurements were performed by one individual during intervention and another 
Table I. Coronary Angiography and Intravascular Ultrasound Parameters

\begin{tabular}{lccc}
\hline & Prestent & Poststent & $P$ \\
\hline Angiographic & & & \\
Reference lumen diameter & $3.45 \pm 0.5$ & $3.45 \pm 0.5$ & NS \\
Minimal lumen diameter & $1.5 \pm 0.45$ & $3.5 \pm 0.45$ & $<0.001$ \\
\% stenosis & $78 \pm 16$ & 0 & \\
& & & \\
LAD (\% stenosis) & $79 \pm 17$ & 0 & \\
Cx (\% stenosis) & $75 \pm 19$ & 0 & NS \\
RCA (\% stenosis) & $78 \pm 18$ & 0 & $<0.0001$ \\
IVUS & & & $<0.0001$ \\
RLA (mm ${ }^{2}$ ) & $9.7 \pm 2.7$ & $9.7 \pm 2.7$ & $<0.0001$ \\
TLLA (mm $\left.{ }^{2}\right)$ & $1.8 \pm 0.5$ & $8.5 \pm 0.5$ & \\
LAS \% & $0.82 \pm 12.3$ & $0.14 \pm 27$ & \\
CTFC & $35.3 \pm 16.8$ & $16.9 \pm 4.3$ & \\
\hline
\end{tabular}

$\mathrm{LAD}=$ left anterior descending, $\mathrm{RCA}=$ right coronary artery; $\mathrm{Cx}=$ circumflex artery; IVUS $=$ intra vascular ultrasound; TFC $=$ TIMI frame count CTFC $=$ corrected TIMI frame count; RLA = refe rence lumen area; TLLA = target lesion lumen area.

observer who was blinded to the clinical and angiographic information. In the case of a disagreement, a consensus was reached in a third session.

The following criteria were used in the assessment of optimal stent procedures: (1) intrastent area/mean reference lumen area $\geq 0.80$ and/or intrastent area $>7 \mathrm{~mm}^{2}$, and (2) symmetrical expansion with full apposition of the stent to the vessel wall. ${ }^{11-13)}$ In the case of suboptimal IVUS criteria, further balloon inflations were performed until optimal criteria were achieved.

Patients with a de novo lesion with $>50 \%$ stenosis or flow-limiting dissection proximal or distal to the target lesion, or distal no-reflow, were excluded from the study.

Statistical analysis: The continuous variables are presented as means \pm SD. Continuous variables were compared using Student's $t$-test, and categorical variables were compared using the chi-square test. A $P$ value $<0.05$ was accepted as statistically significant. The correlations between the CTFC and IVUS measurements were assessed using linear regression analysis.

\section{RESULTS}

The diameters of the reference lumen and target lesions, angiographic \% stenosis, IVUS-derived planimetric measurements and luminal \% area stenosis before and after stenting are presented in Table I. Mean target lesion lumen areas 
were $1.8 \pm 0.5 \mathrm{~mm}^{2}$ and $8.5 \pm 0.5 \mathrm{~mm}^{2}(P<0.0001)$, and luminal $\%$ area stenosis was $82 \pm 12.3 \%$ and $14 \pm 27 \%(P<0.0001)$ before and after intracoronary stenting, respectively. Acute luminal gain was $6.65 \pm 1.2 \mathrm{~mm}^{2}$. Pre- and postintervention CTFC were $35.3 \pm 16.8$ and $16.9 \pm 4.3$, respectively $(P<0.0001)$.

Linear regression analysis performed on a total of 76 IVUS studies showed that CTFC had a good and negative correlation to target lesion lumen area $(r=$ $-0.70, P<0.001$ ) (Figure 1), and a positive correlation to luminal \% area stenosis $(r=0.69, P<0.001)$ (Figure 2). There was a modest but significant correlation

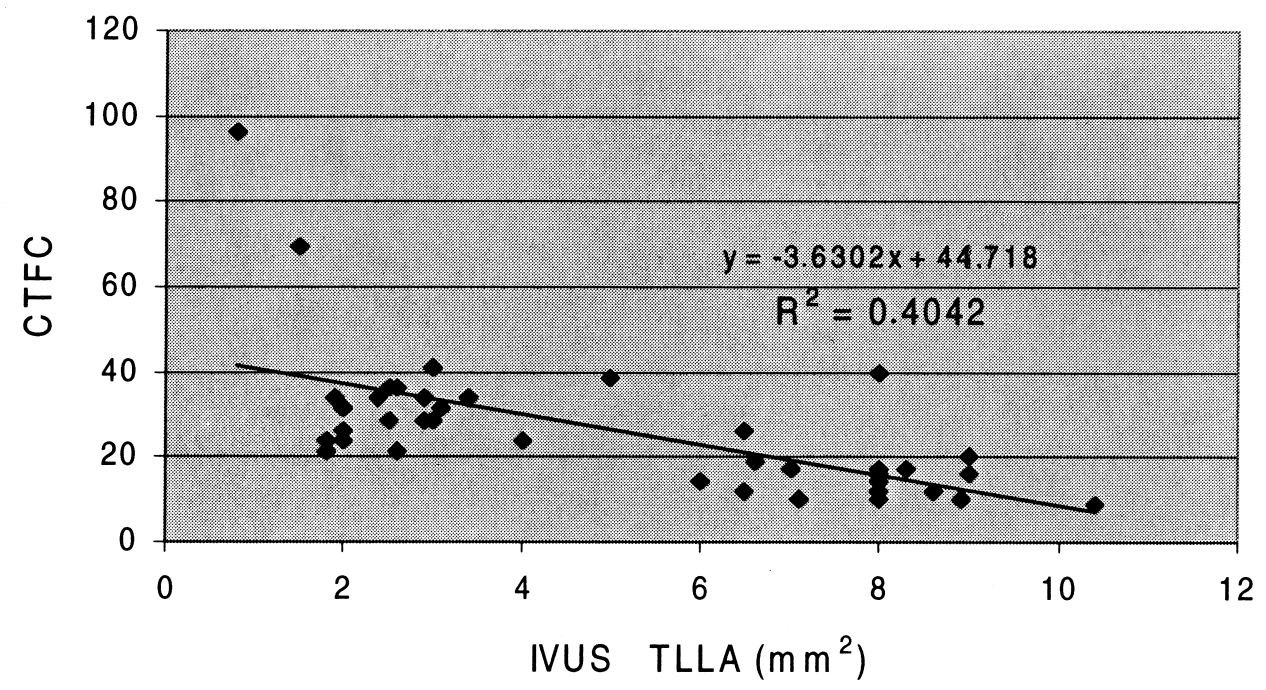

Figure 1. Correlation between IVUS target lesion lumen area (TLLA) and corrected TIMI frame count (CTFC).

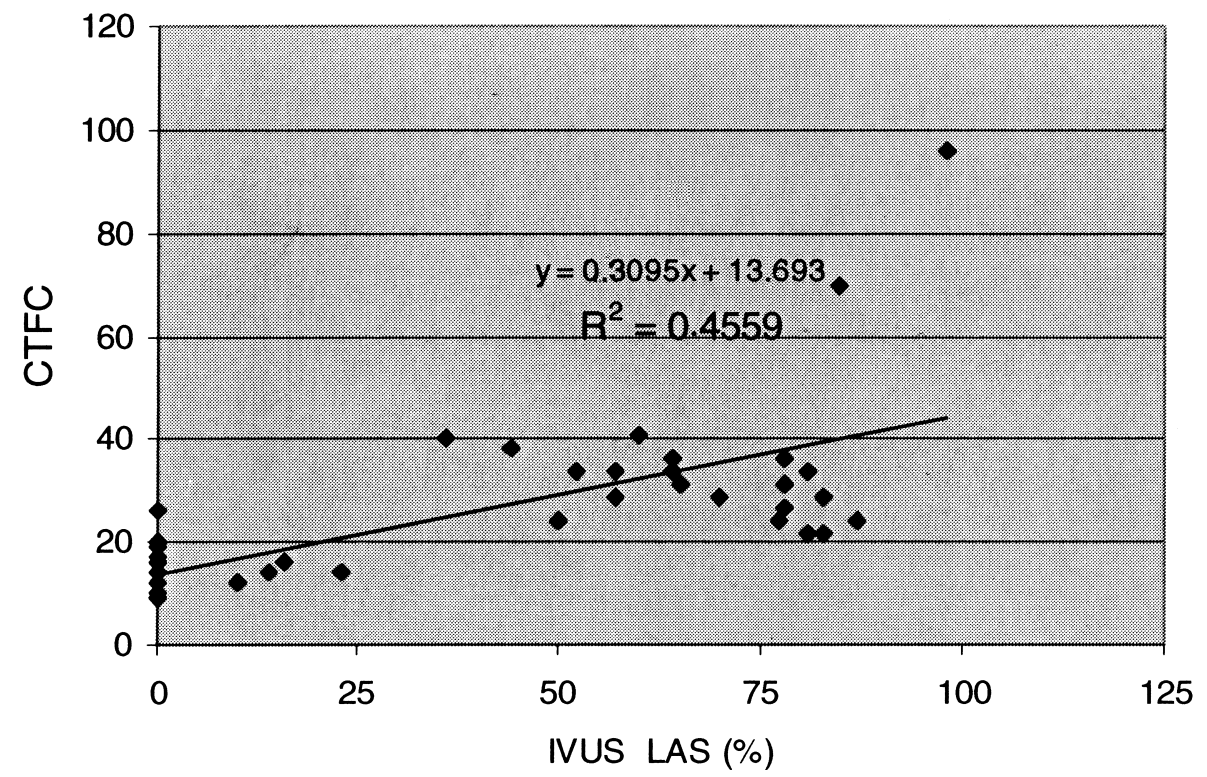

Figure 2. Correlation between luminal area stenosis (LAS) and corrected TIMI frame count (CTFC). 
between acute luminal gain and postprocedural CTFC change $(r=0.5, P<0.05)$ (Figure 3).

Patients with acute coronary syndrome and stable coronary artery disease were compared with respect to pre- and postintervention IVUS parameters and CTFC (Table II). Before stenting, patients with acute coronary syndrome had a lower target lesion lumen area $\left(1.3 \pm 0.4 \mathrm{~mm}^{2}\right.$ vs $\left.1.9 \pm 0.3 \mathrm{~mm}^{2}, P>0.05\right)$, a higher luminal $\%$ area stenosis $(85 \pm 9.2 \%$ vs $78 \pm 11.3 \%, P>0.05)$, and a higher

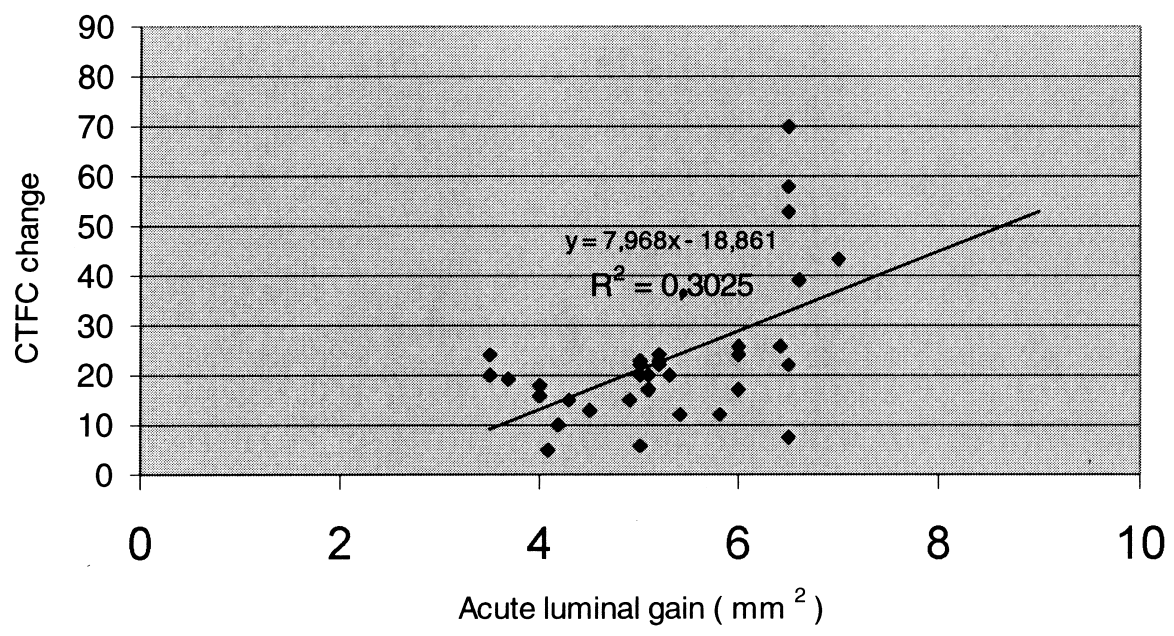

Figure 3. Correlation between acute luminal gain $\left(\mathrm{mm}^{2}\right)$ and corrected TIMI frame count (CTFC) change.

Table II. Parameters of Coronary Angiography and IVUS in Patients with Acute Coronary Syndrome and Stable Coronary Artery Disease

\begin{tabular}{|c|c|c|c|c|c|c|}
\hline & \multicolumn{3}{|c|}{ Prestent } & \multicolumn{3}{|c|}{ Poststent } \\
\hline & $\mathrm{ACS}$ & SAD & $P$ & $\mathrm{ACS}$ & SAD & $P$ \\
\hline \multicolumn{7}{|l|}{ Angiographic } \\
\hline Reference lumen diameter & $3.66 \pm 0.5$ & $3.43 \pm 0.6$ & NS & $3.66 \pm 0.5$ & $3.43 \pm 0.6$ & NS \\
\hline Minimal lumen diameter & $1.1 \pm 0.4$ & $1.6 \pm 0.3$ & NS & $3.6 \pm 0.5$ & $3.4 \pm 0.4$ & NS \\
\hline$\%$ stenosis & $79 \pm 17$ & $73 \pm 16$ & NS & 0 & 0 & \\
\hline \multicolumn{7}{|l|}{ IVUS } \\
\hline $\operatorname{RLA}\left(\mathrm{mm}^{2}\right)$ & $9.8 \pm 2.5$ & $9.6 \pm 2.8$ & NS & $9.7 \pm 2.7$ & $9.6 \pm 2.8$ & NS \\
\hline $\operatorname{TLLA}\left(\mathrm{mm}^{2}\right)$ & $1.3 \pm 0.4$ & $1.9 \pm 0.3$ & NS & $8.2 \pm 0.8$ & $8.3 \pm 0.7$ & NS \\
\hline LAS $\%$ & $0.85 \pm 9.2$ & $0.78 \pm 11.3$ & NS & $15 \pm 23$ & $16 \pm 18$ & NS \\
\hline CTFC & $37 \pm 14.8$ & $34 \pm 13.2$ & NS & $18.2 \pm 5.2$ & $15 \pm 4.2$ & NS \\
\hline Net TLLA change & & & & $7.1 \pm 1.5$ & $6.4 \pm 1.6$ & NS \\
\hline Net CTFC change & & & & $18.4 \pm 5.5$ & $20 \pm 6.3$ & NS \\
\hline
\end{tabular}

$\mathrm{LAD}=$ left anterior descending, $\mathrm{RC}=$ right coronary artery; $\mathrm{Cx}=$ circumflex artery; IVUS = intravascular ultrasound TFC $=$ TIMI frame count $\mathrm{CTFC}=$ corrected TIMI frame count; $\mathrm{RLA}=$ reference lumen area; $\mathrm{TLLA}=$ target lesion lumen area; $\mathrm{ACS}=$ acute coronary syndrome; $\mathrm{SAD}=$ stable coronary artery disease. 
CTFC $(37 \pm 14.8$ vs $34 \pm 13.2, P>0.05)$ compared to those with stable coronary artery disease. However, the difference between the two groups of patients was statistically insignificant. Similar final stent lumen areas and residual luminal $\%$ area stenosis were attained in the two subgroups. In patients with acute coronary syndrome and stable coronary syndrome, final luminal \% area stenosis was $15 \pm 23 \%$ and $16 \pm 18 \%(P>0.05)$, and stent lumen area was $8.2 \pm 0.8 \mathrm{~mm}^{2}$ and $8.3 \pm 0.7 \mathrm{~mm}^{2}(P>0.05)$, respectively. Patients with acute coronary syndrome had a slightly higher post-intervention CTFC (18.2 \pm 5.2 vs $15 \pm 4.2, P>0.05)$ and a lower improvement in CTFC (18.4 \pm 5.5 vs $20 \pm 6.3, P>0.05)$ (Table II).

The left anterior descending artery and circumflex/right coronary arteries were compared with respect to corrected and uncorrected TFC, and the IVUSderived measurements. Angiographic diameters of the reference lumen and target lesions, and mean \% diameter stenosis were not different between the left anterior descending and circumflex/right coronary artery systems either before or after stenting $(P>0.05)$. Neither preintervention nor postintervention IVUS measures were different between the two coronary artery systems $(P>0.05)$ (Figure 4$)$. Similarly, neither the preintervention nor postintervention CTFC were different between the systems $(P>0.05)$. Uncorrected TFC before and after stenting, and the acute change in uncorrected TFC in the different coronary arteries are given in Figure 5. Prior to stenting, uncorrected TFC was significantly higher in the left anterior descending artery than in the circumflex and right coronary arteries $(P<0.05)$. However, no difference in CTFC between these coronary arteries prior

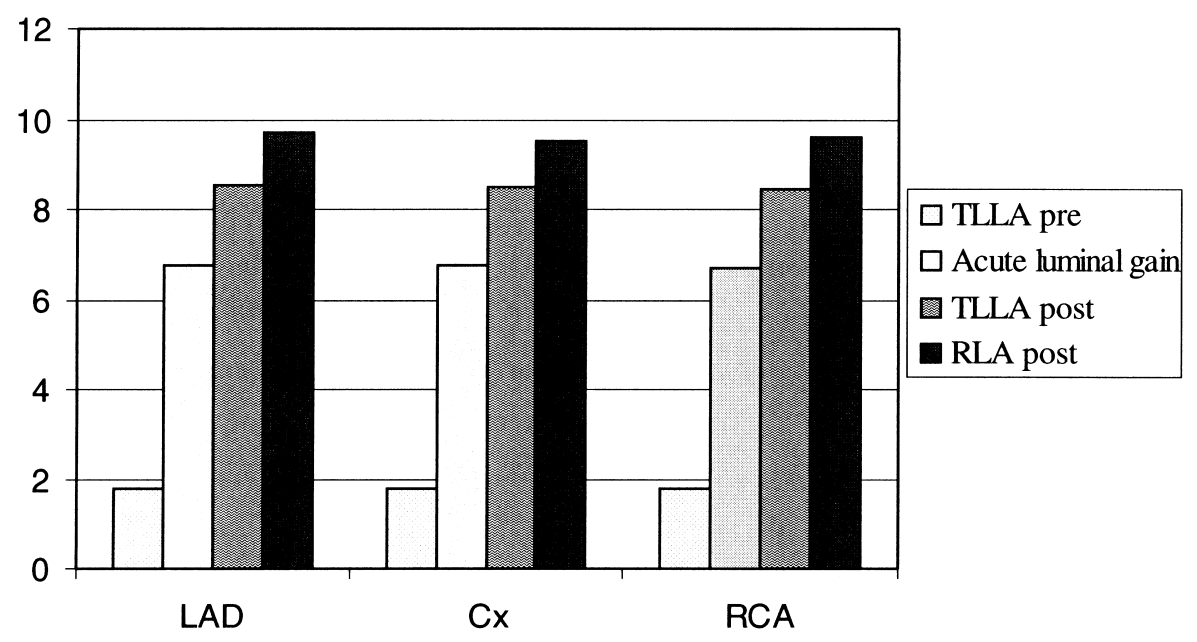

Figure 4. Pre- and postintervention intravascular ultrasound measures $\left(\mathrm{mm}^{2}\right)$. 


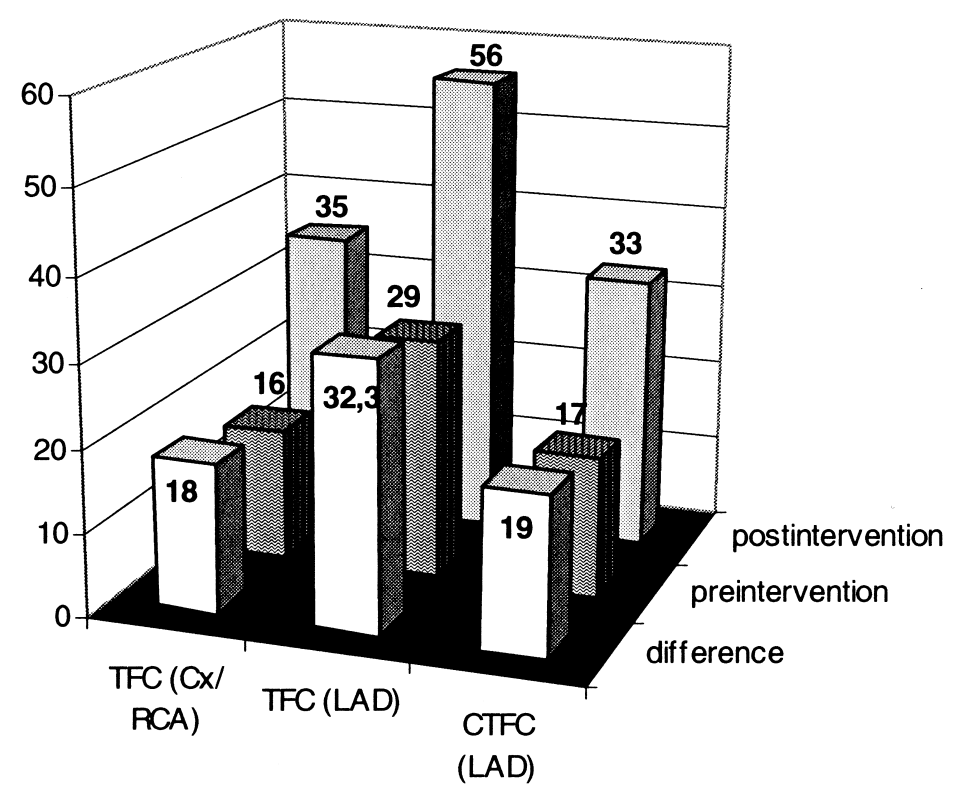

Figure 5. Pre- and postintervention TIMI frame count (TFC) and corrected TIMI frame count (CTFC) according to coronary artery.

to stenting was found $(P>0.05)$. Similar results were also observed with respect to uncorrected and corrected TFC measured in the different arteries after stenting. Postprocedural uncorrected TFC in the left anterior descending artery was significantly higher than that in the circumflex and right coronary arteries $(P<0.05)$, whereas the postprocedural difference between the two coronary artery systems disappeared after correction of TFC $(P>0.05)$ (Figure 5).

\section{DISCUSSION}

The corrected TIMI frame count method has been developed as a continuous index of coronary flow with high intraobserver and interobserver reproducibility. ${ }^{2,14}$ However, there is controversy concerning the variables affecting the CTFC. ${ }^{2,14,15)}$ The distance to the anatomic landmarks, catheter size, the degree of engagement, dye injection rate, the phase of the cycle in which the dye is injected, heart rate, distal resistance to flow, wall motion abnormalities, and viability status of the myocardium have been considered as potential factors which may introduce variability into the TFC. ${ }^{2,14,15)}$ It has been reported that the dye injection rate and catheter size had no effect on TFC whereas nitrate use, heart rate, and the cycle in which the dye is injected had significant effects on TFC. ${ }^{10)}$ It was reported that CTFC varied only a small amount in association with body size, sys- 
temic arterial pressure, age, and sex. ${ }^{12)}$

The vast majority of angiographic studies have investigated the angiographic correlates of TFC, such as lumen and lesion diameters, and \% diameter stenosis. ${ }^{1-3)}$ No correlation has been reported between improvement in CTFC and either the change in the minimum lumen diameter or the change in $\%$ diameter stenosis after thrombolysis. ${ }^{1-3)}$ Edep, et al reported that stenting in acute myocardial infarction was associated with a greater postprocedure luminal diameter and improvement in CTFC than percutaneous coronary angioplasty. ${ }^{8}$ It was reported that CTFC was higher than normal both in the culprit and non-culprit arteries in patients with acute coronary syndromes, and this increase in CTFC has been ascribed to a transient increase in the microvascular resistance. ${ }^{2,16)}$ However, CTFC has not been investigated in patients who underwent elective percutaneous intervention.

Novel techniques based on transmural imaging of the coronary artery wall (ie, intravascular ultrasound) or physiological assessment of coronary stenosis severity and/or flow reserve (ie, fractional flow reserve and intracoronary Doppler) have provided more comprehensive data than those obtained from coronary angiography. ${ }^{17-20)} \mathrm{A}$ minimum luminal area $<4 \mathrm{~mm}^{2}$ measured by IVUS has been reported to predict myocardial ischemia detected by myocardial perfusion scintigraphy. ${ }^{17)}$ Moreover, minimal luminal areas $\geq 4 \mathrm{~mm}^{2}$ and $\geq 6 \mathrm{~mm}^{2}$ have been reported to have high diagnostic accuracy in predicting coronary flow reserves of $\geq 2$ and $\geq 2.5$, respectively. ${ }^{20-21)}$ Pressure-derived myocardial fractional flow reserve has also been developed as a practical method for the functional assessment of coronary stenosis. ${ }^{18,22-25)}$

Although the correlations among the fractional flow reserve, Doppler flow velocimetry, and IVUS parameters have been assessed, to the best of our knowledge, no study has investigated the correlation between CTFC and IVUS-derived planimetric measures. Kern, et al reported a modest but significant correlation between postangioplasty Doppler flow velocity and TIMI grade. ${ }^{19)}$ Manginas, et al proposed an alternative method to measure coronary flow reserve using the baseline and hyperemic TFC, and reported a good correlation between the net change in TFC and coronary flow reserve calculated by Doppler Flowire. ${ }^{6}$

Takagi, et al reported that the myocardial fractional flow reserve was closely correlated to area stenosis (\%) and minimal target lesion area measured by IVUS, and that the combination of minimal target lesion lumen area $\left(<3 \mathrm{~cm}^{2}\right)$ and area stenosis $(>0.60)$ criteria was able to predict impaired $(<0.75)$ myocardial fractional flow reserve. ${ }^{23)}$ However, Fearon, et al reported that optimal $(\geq 0.96)$ myocardial fractional flow reserve after stenting was not reliable for predicting the optimal IVUS criteria of stent expansion, whereas impaired $(<0.96)$ myocardial fractional flow reserve indicated suboptimal stent lumen area measured by 
IVUS. ${ }^{24)}$ Recently, Umman, et al reported that the improvement in CTFC after stenting was closely correlated to the difference between pre- and postintervention myocardial fractional flow reserve. $\left.{ }^{25}\right)$

The present study demonstrated that CTFC had a good negative correlation to target lesion lumen area, and a good correlation to luminal \% area stenosis. Acute luminal gain in the culprit lesion due to stenting showed a modest but significant correlation to postprocedural improvement in the CTFC. This may be associated with a persistence of increased distal resistance due to microvascular no-reflow and/or non-viable myocardium despite the optimal stenting in patients with acute coronary syndrome. Subgroup analysis revealed that patients with acute coronary syndrome had a lower preintervention target lesion lumen area, and a higher CTFC as compared to patients with stable angina pectoris. Although postintervention target lesion lumen area was similar between the two subgroups, patients with acute coronary syndrome had a higher postintervention CTFC, a lower luminal gain, and a lower improvement in CTFC than patients with stable coronary arteries. However, the differences between the patients with acute coronary syndrome and stable coronary artery disease were not statistically significant. We also tested the validity of correction by comparing the CTFC of the different coronary arteries with comparable angiographic and IVUS-derived characteristics. Moreover, another comparison between uncorrected and corrected TFC was also performed. Preintervention CTFC and IVUS measurements were not different between the left anterior descending artery and circumflex/ right coronary arteries. The similarity between the two different coronary artery territories with respect to CTFC and IVUS parameters was preserved after optimal stenting. However, in the absence of the correction procedure performed by dividing the TFC of the left anterior descending artery by 1.7, the left anterior descending artery had a significantly higher TFC than the circumflex/right coronary arteries in both the pre- and postintervention period. Again, a significant difference between the uncorrected TFC and CTFC of the left anterior descending artery was also detected in the postintervention period. The lack of a difference between the CTFC of the different coronary arteries with comparable IVUS parameters, and the presence of a significant difference between the CTFC and uncorrected TFC of the left anterior descending coronary artery are considered to be further support for the validity of the correction procedure.

Study limitations: The assessment of CTFC did not take into consideration the left ventricular wall motion abnormalities and viability status of myocardium related to culprit arteries. Moreover, the relationships between CTFC and other functional data which might be provided by videodensitometric coronary flow, myocardial fractional flow reserve, or Doppler Flowire, were not investigated. 
In summary, this study correlated the CTFC to IVUS-derived planimetric measurements of lesion lumen area and \% area stenosis in culprit arteries of patients who underwent stenting. However, the correlation between an improvement in CTFC and acute luminal gain measured by IVUS was found to be modest. The results from different coronary arteries with comparable IVUS parameters seem to support the validity of the correction procedure in TIMI frame count.

\section{REFERENCES}

1. TIMI Study Group. The Thrombolysis in Myocardial Infarction (TIMI) trial. N Engl J Med 1985; 31: 932-6.

2. Gibson CM, Cannon CP, Daley WL, et al. TIMI frame count: a quantitative method of assessing coronary artery flow. Circulation 1996; 93: 879-88.

3. Gibson CM, Ryan KA, Kelley M, et al. Methodological drift in the assessment of TIMI grade 3 flow and its implications with respect to the reporting of angiographic trial results. The TIMI Study Group. Am Heart J 1999; 6: 1179-84.

4. French JK, Straznicky IT, Webber B, et al. Angiographic frame counts 90 minutes after streptokinase predict left ventricular function at 48 hours following myocardial infarction. Heart 1999; 2: 128-33.

5. French JK, Hyde TA, Straznicky IT, Andrews J, et al. Relationship between corrected TIMI frame counts at three weeks and late survival after myocardial infarction. J Am Coll Cardiol 2000; 6: 1516-24.

6. Manginas A, Gatzov P, Chasikidis C, Vaudris V, Pavlides G, Cokkinos DV. Estimation of coronary flow reserve using the thrombolysis in myocardial infarction (TIMI) frame count method. Am J Cardiol 1999, 83: 1562-5.

7. Stankovic G, Manginas A, Vaudris V, et al. Prediction of restenosis after coronary angioplasty by use of a new index TIMI frame count /Minimal luminal diameter ratio. Circulation 2000; 101: 962-8.

8. Edep ME, Guarneri EM, Teirstein PS, et al. Differences in TIMI frame count following successful reperfusion with stenting or percutaneous transluminal coronary angioplasty for acute myocardial infarction. Am J Cardiol 1999; 83: 1326-9.

9. Mintz GS, Painter JA, Pichcard AD, et al. Atherosclerosis in angiographically "normal” coronary artery referance segments : an intravascular ultrasound study with corrections. J Am Coll Cardiol 1995; 25: 1479-85.

10. Mintz GS, Nissen SE, Anderson WD, et al. American College of Cardiology Clinical expert consensus document on standards for acquisition, measurement and reporting of intravascular ultrasound studies (IVUS). A report of the American College of Cardiology Task Force on Clinical Expert Consensus Documents. J Am Coll Cardiol 2001; 5: 1478-92.

11. Moussa I, Moses J, Di Mario C, et al. Does the specific intravascular ultrasound criterion used to optimize stent expansion have an impact on the probabilitiy of stent restenosis. Am J Cardiol 1999; 83: 1012-7.

12. Nissen SE, Yock P. Intravascular ultrasound : Novel pathopysiological insights and current clinical applications. Circulation 2001; 103: 604-16.

13. de Jaegere P, Mudra H, Figulla H, et al. Intravascular ultrasound-guided optimised stent deployment: Immediate and 6 months clinical and angiographic from the Multicenter Ultrasound Stenting in Coronaries Study (MUSIC study). Eur Heart J 1998; 19: 1214-23.

14. Abaci A, Oguzhan A, Eryol N.K, Ergin A. Effects of potential confounding factors on the thrombolysis in myocardial infarction (TIMI) trial frame count and its reproducibility. Circulation 1999; 100: 2219-23.

15. Faile BA, Guzzo JA, Tate DA, Nichols TC, Smith SC, Dehmer GJ. Effects of sex, hemodynamics, body size, and other clinical variables on the corrected thrombolysis in myocardial infarction frame count used as an assessment of coronary blood flow. Am Heart J 2000; 2: 308-14.

16. Gibson CM, Ryan KA, Murphy SA, et al. Impaired coronary blood flow in nonculprit arteries in the setting of acute myocardial infarction. The TIMI study group. Thrombolysis in myocardial infarction. J Am Coll Cardiol 1999; 34: 974-82. 
17. Nishioka T, Amanullah AM, Berlung H, et al. Clinical validation of intravascular ultrasound imaging for assessment of coronary stenosis severity: Comparison with stress myocardial perfusion imaging. J Am Coll Cardiol 1999; 33: 1870-8.

18. Hanekamp C, Koolen J, Pijls N, Michels HR, Bonnier HJRM. Comparison of quantitative coronary angiography, intravascular ultrasound, and coronary pressure measurement to assess optimum stent deployment. Circulation 1999; 99: 1015-21.

19. Kern MJ, Dupouy P, Drury JH, et al. Role of coronary artery lumen enlargement in improving coronary blood flow after balloon angioplasty and stenting: A combined intravascular ultrasound Doppler flow and imaging study. J Am Coll Cardiol 1997 29: 1520-7.

20. Abizaid A, Mintz GS, Pichcard AD, et al. Clinical, intravascular ultrasound, and quantitative angiographic determinants of coronary flow reserve before and after percutaneous transluminal coronary angioplasty. Am J Cardiol 1998; 82: 423-8.

21. Serruys PW, di Mario C, Piek J, et al. Prognostic value of intracoronary flow velocity and diameter stenosis in assessing the short- and long-term outcomes of coronary balloon angioplasty: the DEBATE Study (Doppler Endpoints Balloon Angioplasty Trial Europe). Circulation 1997 Nov 18; 96: 3369-77.

22. Pijls NHJ, Van Gelder B, Van der Voort P, et al. Fractional flow reserve. A useful index to evaluate the influence of an epicardial coronary stenosis on myocardial blood flow. Circulation 1995; 92: 3183-93.

23. Takagi A,Tsurumi Y, Ishii Y, Suzuki K, Kawana M, Kasamaki H. Clinical potential of intravascular ultrasound for physiological assessment of coronary stenosis. Relationship between quantitative ultrasound tomography and pressure-derived fractional flow reserve. Circulation 1999; 100: 250-5.

24. Fearon WF, Luna J, Samady H, et al. Fractional flow reserve compared with intravascular ultrasound guidance for optimizing stent deployment. Circulation. 2001; 104: 1917-22.

25. Umman B, Nisanci Y, Sezer M, et al. The relationship between corrected TIMI frame count and myocardial fractional flow reserve. J Invasive Cardiol 2002; 14: 125-8. 